\title{
LII. On the recent alterations said to be made by some tuners of musical instruments, in the places of the wolves, or largely tempered concords, on common 12-stringed or Douzeave Keyed- Instruments. With some queries thereon, to Musicians
}

\section{Mr. John Farey Senior}

To cite this article: Mr. John Farey Senior (1820) LII. On the recent alterations said to be made by some tuners of musical instruments, in the places of the wolves, or largely tempered concords, on common 12-stringed or Douzeave Keyed-Instruments. With some queries thereon, to Musicians, Philosophical Magazine Series 1, 56:271, 341-342, DOI: $\underline{10.1080 / 14786442008652418}$

To link to this article: http://dx.doi.org/10.1080/14786442008652418

Published online: 23 Jul 2009.

Submit your article to this journal $\sqsubset$

山ll Article views: 2 


\section{[341]}

L11. On the recent Alterations said to be made by some Tuners of Musical Instruments, in the Places of the Wolves, or largely tempered Concords, on common 12-stringed or Douzeave Keyed-Instruments. Wilh some Queries thereon, to Musicians.

By Mr. Johr FaRey Senior.

\section{To Mr.Tilloch.}

Sir, $-T_{\text {B N years ago I drew up and communicated through }}$ your pages*, Six Musicas Theorems, showing the various relations of the Temperaments, of all the 72 concords capable of being taken on a Douzeave Instrument, or one having only 12 Notes in the Octave; and soon after, Fifteen Musical Corollaries, derived from those Theorems, were also given in your Work $\uparrow:$ at this period, it was the general opinion of the professional Tuners and scientific Musicians whom I had opportunities of consulting, that it was usual and proper, to consider the five short Fingerkeys of Instruments, as producing the Notes F* $\mathrm{C}$, $\mathrm{G}_{*} ; \mathrm{Eb}$ and $\mathrm{Bb}$; and the resulting Fifth or Quint Wolf to lie, between $\mathrm{G}$ and $\mathrm{Eb}$ : since then, the arrangement of the Pedals of the improved Instruments made by Mr. Loeschman, and for Mr. Liston, and the excellent Work of the latter (entitled "An Essay on perfect Intonation,") have shown, that these ingenious Individuals considered, the common or original Scale, or that which would be found by using the twelve ordinary Finger-keys on their respective Instruments (none of their Pedals were in action) to consist of the seven natural Notes, and of those three sharpened and two flattened Notes, above mentioned: and every thing contained in my Papers alluded to, relative to the places in the Scale, in which the several Wolves, or resulting, and mostly also the largest tempered Concords, were to be found, depended on this assumption, viz. of it being the practice of Tuners, to effect their tuning, upwards by the Tempered Fifths $C G, G D, D A, A E, E B, B F * F * C *$, and $C * G *$, and downwards by the similar Fifths $\mathrm{cF}, \mathrm{FBb}$, and $\mathrm{Bb} \mathrm{Eb}$, so as to meet in the resulting or Wolf Fifth $G_{*} E b$ : such however appears now, not to be the invariable practice of Piano-Forte Tuners; since the Rev. C. J. Smyth, of Norwich, has informed me, that several Tuners for Mr. Wornam, of Wignore-street, and other makers of Piano-Fortes in the newest fashion, are in the habit, of laying the Bearing as they sometimes call it, or throwing the Quint Wolf, between the Notes $C$ and $A b$; or in other words, their Scale is made to contain, seven natural, two sharpened and three

* See P. M. vol. xxxyi. p. 39.

+ P. M. vol. xxxvi. p. 374.

flattened 


\section{Queries respecting Tuning of Musical Instruments.}

flattened Notes, instead of those assumed in my Theorems and Corollaries; which they effect, by stopping in their upward series of consecutive tempered Fifths, with $C_{*}$; and continuing their downward series of similar Fifths, one note further than is mentioned above, viz. to Ab.

The magnitudes and proportions of the several Temperaments and Wolves (although not their places) will still all be truly represented by my Theorems or Corollaries, in this new manuer of laying the Quint Wolf, or in any other, which the convenience of Musicians, or the fancies of Tuners, may suggest ; provided only, that eleven of the Fifths out of the twelve, are precisely equal in magnitude. In either of two particular cases of equally Tempered Fifths*, my Theorems will still apply, viz. first, in the Isotonic or common equal-temperament Scale, wherein each of the twelve Fifths is flattened the same quantity (or 1-12th of the Diaschisma, $12 \Sigma+m \dagger)$; and second, the Scale wherein each of the twelve temperaments of the Fifths are equal, (each 1-10th of the Diaschisma) yet eleven of them are flat, and one sharp, viz. G* Eb.

I have ventured to call the attention of your Musical Readers to this subject, in order to request to be informed, through the medium of your pages, or otherwise :

1st. Whether this method of laying the Quint Wolf on $C_{\text {, }}$ rather than on $\mathrm{G} *$, has yet prevailed to any considerable extent?

2nd. Whether, for the general run of Piano-Forte Music, it be really an Improvement to make this change, or otherwise?

3rd. Whether either of the three Systems of equal Fifiths, hut some of them sharpened, which are mentioned herein, have been tried?: and if so, how were they approved?

\section{I am your obedient servant,}

37, Howland-street, Fitzroy-square, Oct. 17,1820 .

JoHN FAREX Sen.

- As the amusement of occasional leisure Hours, I have considered and calculated the Intervals of some other equal Temperaments of the Fifths, to which my Theorems above quoted, will not apply; in one of these, the Fifth Temperament is $\frac{1}{8}$ th of the Diaschisma (or $1 \cdot 5 \bar{\Sigma}+\frac{1}{8} \mathrm{~m}$ ), this being the sharp Temperament of two of the Fifths, viz. $C$ and $G$ Eb, while all the 10 other Fifths are flattened the same quantity. In another of these Systems, th of the Diaschisma (or $2 \Sigma+\frac{1}{6} m$ ) is the sharp Temperament of thi ee of the Fiftls, viz. $F * C * C * G *$ and $G * E b$, while the 9 other Fifths are as much flattened.

+ See P. M. vol. xxviii. Plate V. p. 140. 